TITLE:

\title{
Magnetic field distribution control with anisotropic inter-pole magnetic field
}

$\operatorname{AUTHOR}(S)$ :

Iwashita, Y; Arimoto, Y; Sato, A

\section{CITATION:}

Iwashita, Y ... [et al]. Magnetic field distribution control with anisotropic inter-pole magnetic field. IEEE TRANSACTIONS ON APPLIED SUPERCONDUCTIVITY 2006, 16(2): 12861289

ISSUE DATE:

2006-06

URL:

http://hdl.handle.net/2433/50332

\section{RIGHT:}

(c)2006 IEEE. Personal use of this material is permitted. However, permission to reprint/republish this material for advertising or promotional purposes or for creating new collective works for resale or redistribution to servers or lists, or to reuse any copyrighted component of this work in other works must be obtained from the IEEE. 


\title{
Magnetic Field Distribution Control With Anisotropic Inter-Pole Magnetic Field
}

\author{
Yoshihisa Iwashita, Yasushi Arimoto, and Akira Sato
}

\begin{abstract}
A novel method to control a magnetic field distribution in a magnet is devised with use of inter-poles made of anisotropic magnet material. One can generate a gradient field in a constant gapped magnet with this technique; the effective length of the magnet can be constant, independent of the entrance position. Such a magnet can have not only constant gap but also smaller fringing field compared with a conventional one that controls its field distribution by changing the gap height.
\end{abstract}

Index Terms-Accelerator magnets, magnetic anisotropy, magnetic circuits, optimization methods.

\section{INTRODUCTION}

$\mathbf{M}$ AGNETS for a combined function synchrotron [1], [2] or FFAG [3] need to generate gradient fields in large gaps. Such field gradients are usually generated by changing their gap distances (magnetic resistances) along the transverse direction with one set of excitation coils (constant magnetomotive force) or by changing the magnetomotive force using trim coils in a constant gap. This paper presents a method to generate such gradient field with a constant gap distance.

The coordinate system used in this paper is the cylindrical one, which is usually used in circular machines namely RSZ plane (R: transversal, S: longitudinal, Z: vertical).

\section{INTER-POLE IN A MAGNET}

Magnets with gradient field (indexed magnets) usually have varying gap distances in accordance with the field to be generated. (see Fig. 1). Taking a line integral along the flux line, the magnetic flux density $B$ at gap distance $g$ with excitation current $I$ is given by $B=\mu_{0} I / g$ when the permeability $\mu$ in the iron is large enough. This enables us to control the magnetic field distribution by changing $g$. This configuration has an advantage because it needs only one set of coils and one power supply. The gap distance variation, however, will break the uniformity of the effective length for such a gradient magnet. Furthermore, the extent of the fringing field may become large at the large gap side when the field level ratio is high, which may interfere with other magnetically sensitive accelerator components nearby such as accelerating cavities with magnetic core load and/or Q-magnets. When we divide the pole into two sections (a flat square block $a$ and the other part b) and switch their positions as shown in Fig. 2, the gap distance in the vicinity of

Manuscript received September 19, 2005.

Y. Iwashita is with the Institute for Chemical Research, Kyoto University, Uji, Kyoto, Japan (e-mail: iwashita@kyticr.kuicr.kyoto-u.ac.jp).

Y. Arimoto and A. Sato are with the Osaka University, Osaka, Japan.

Digital Object Identifier 10.1109/TASC.2005.864300

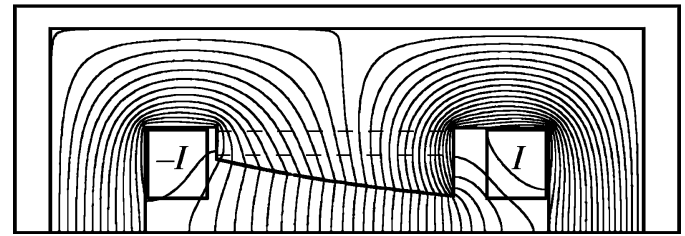

Fig. 1. A magnet with gradient field.

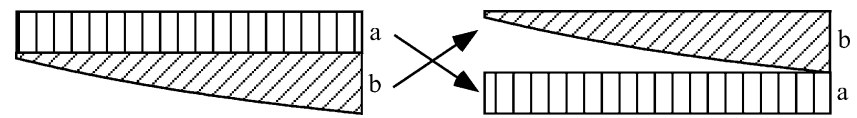

Fig. 2. Divided magnet pole parts are switched.

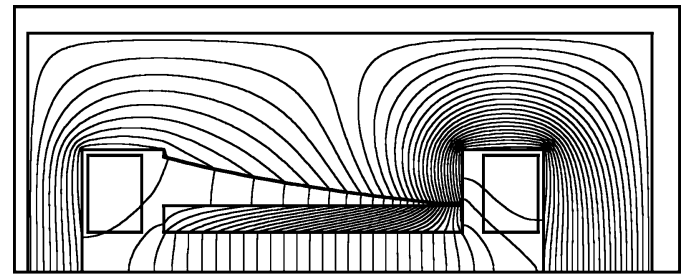

Fig. 3. A magnet with inter-pole made of solid iron.

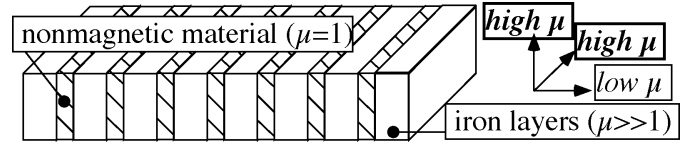

Fig. 4. Composed anisotropic material.

the median plane becomes constant while the total air gap length at a horizontal position does not change. Unfortunately, the solid iron (isotropic material) surface becomes an equipotential surface; the magnetic field in the gap region becomes flat as shown in Fig. 3.

This situation can be resolved by introducing anisotropic material as the inter-pole that has a high permeability in a direction normal to the median plane and low permeability in the direction parallel to the field variation. Such material can be composed by a stack of high permeability iron sheets with nonmagnetic spacers in between (see Fig. 4). The equivalent permeability $\mu_{\mathrm{e}}$ in the stacking direction is given by $\mu_{e}=1 /\left(1-p+p / \mu_{n}\right)$ where $p$ is the packing factor and $\mu_{\mathrm{n}}$ is the permeability of the iron sheet. Usually $\mu_{n}$ is high enough that $\mu_{\mathrm{e}}$ can be rewritten as $\mu_{e}=1 /(1-p)$. When the packing factor $\mathrm{p}$ is 0.667 , the $\mu_{\mathrm{e}}$ becomes only 3 , while those of the other directions remain high. The resulting magnetic field distribution is shown in Fig. 5. Because the horizontal permeability is not unitary, the generated field gradient becomes slightly smaller than that calculated from the sum of the length of two gaps: upper gap and median gap. 


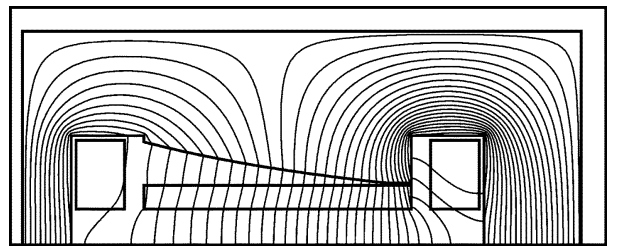

Fig. 5. Flux plot of a magnet with a set of inter-poles made of anisotropic magnet material that has low permeability $(\mu \sim 3)$ in horizontal direction.

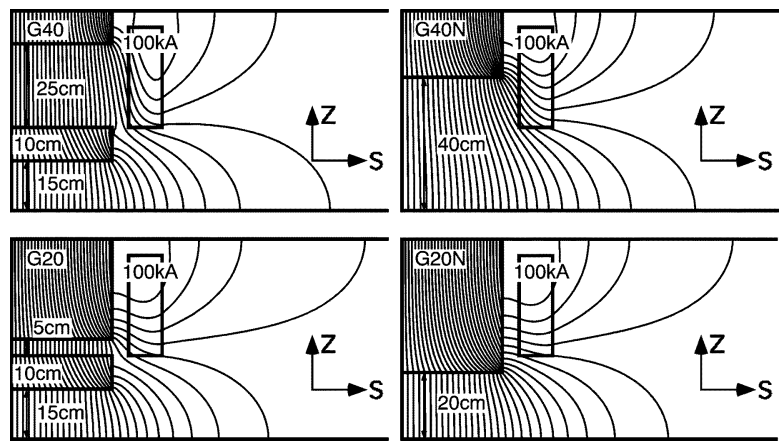

Fig. 6. Magnet configurations to evaluate the effective lengths: the magnets with inter-poles (left) and the conventional ones (right). The upper and bottom figures represent weaker and stronger configurations, respectively.

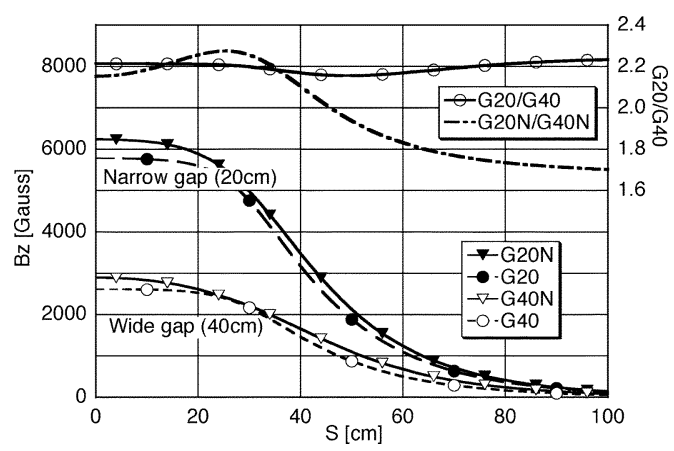

Fig. 7. The magnetic field distributions (fringing field) of large and small gapped magnets as a function of longitudinal coordinate. Use of the inter-poles keeps the ratio fairly constant until the absolute value become less significant.

Strictly speaking, the actual number of gaps in a real magnet is three, while we are considering only the upper half of a magnet. There is one drawback, that the low packing factor decreases the allowable magnetic flux density. This will be revisited later.

\section{EFFeCtive LeNGTH OF A MAGNET}

The effects of the inter-pole on the fringing field are evaluated by pole geometries with different total gap distance (see Fig. 6). Because the pole width is usually much wider than the gap height as shown in Fig. 5 and thus the variation in transverse direction is smaller than that in longitudinal (S-) direction, 2D calculations substitute the $3 \mathrm{D}$ ones. The left figures show the magnets with inter-poles, while the right figures are the conventional magnets. Both cases have the same total gap distances $(20 \mathrm{~cm}$ and $40 \mathrm{~cm}$ ) to generate the same levels of magnetic field. Fig. 7 shows the field distribution, where the broken lines (G40N and G20N) show magnetic field distributions without inter-poles and the solid lines (G40 and G20) show those with
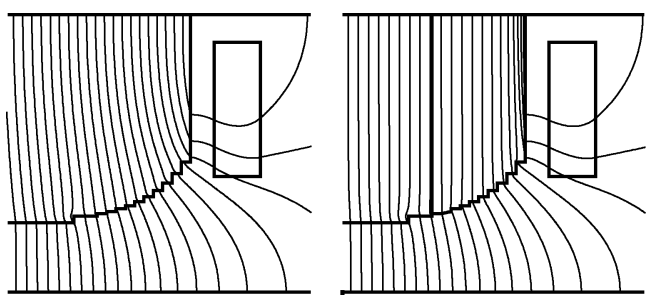

Fig. 8. Flux distributions at the magnet edges of a solid magnet (left) and a laminated magnet (right).

inter-poles. The ratios of magnetic fields for small gap to those for large gap in both cases are also shown. When the inter-poles are used the ratio is kept fairly constant until the absolute values become less significant. The effective length was calculated by taking an integral of $\mathrm{Bz}$ along the horizontal axis and normalized by its maximum value. Then the effective length ratio was obtained by taking the ratio of these two values: with large gap width and with narrow gap width. The ratio for the conventional magnet was evaluated as follows

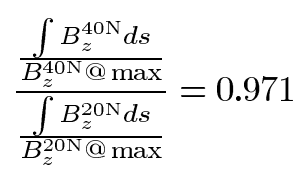

where $B_{z}^{40 \mathrm{~N}}$ and $B_{z}^{20 \mathrm{~N}}$ are the $B_{z}$ components on the median planes for magnets with $40 \mathrm{~cm}$ and $20 \mathrm{~cm}$ gap, respectively. The value for the magnet with inter-pole was calculated as follows

$$
\frac{\frac{\int B_{z}^{40} d s}{B_{z}^{40} @ \max }}{\frac{\int B_{z}^{20} d s}{B_{z}^{20} @ \max }}=1.003 .
$$

These show that the effective length can be well kept constant even with very big change in the gap distance.

\section{CONSIDERATION ON EDDY CURRENT LOSS}

Suppose that the anisotropic material is used at the end of the magnet as shown in Fig. 8. Conventional magnets made of silicon steel sheets, which are usually used in AC magnets, already have this kind of layered configuration. In such a configuration, the flux density at the outer most layers becomes high because of the low permeability normal to the sheet, even with a Rogowskii shape. This flux concentration becomes worse for a sharp edge magnet. Because the low permeability may reduce the flux component penetrating the steel sheets, the eddy current loss may be reduced, which has been cured by cutting slits on the sheets. The high flux density at the outermost layer, however, is likely to cause serious saturation loss. There may be caution needed for such layers in high power application.

\section{Pole Shape AND TRIM CoIL}

Because the permeability of the inter-pole in the horizontal direction is slightly larger than that of vacuum, it filters out local irregularities in the magnetic field distribution. Thus the complex shaped surface may be approximated by a stepwise shape as shown in Fig. 9. The stepwise shape is an exaggerated example 


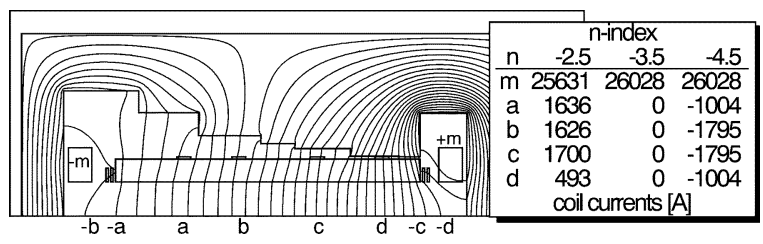

Fig. 9. A magnet with inter-pole that has stepwise approximated iron surface. This example has also trim coils to change the field index. After the step pole shape was optimized for $\mathbf{n}=-3.5$ without trim coils, the positions of four coils together with the currents of the two groups are adjusted to raise the gradient to $\mathrm{n}=-4.5$. Finally, all six currents are adjusted to flatten it to $\mathrm{n}=-2.5$.

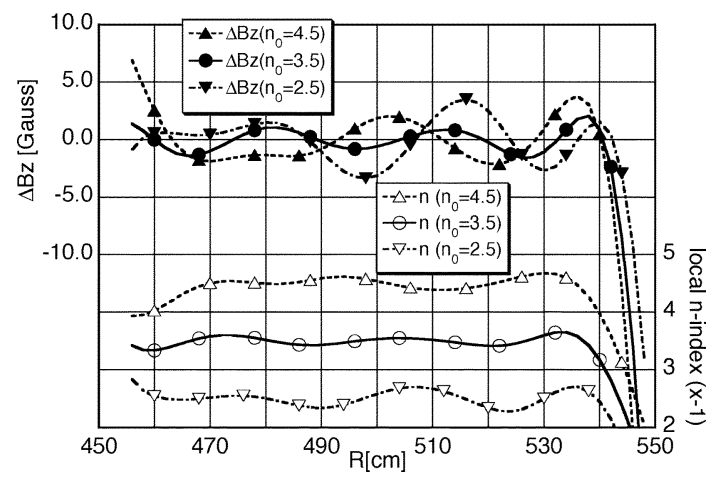

Fig. 10. Field distributions with currents on trim coils. The field index is raised to 4.5 or reduced down to 2.5 from the original value 3.5 .

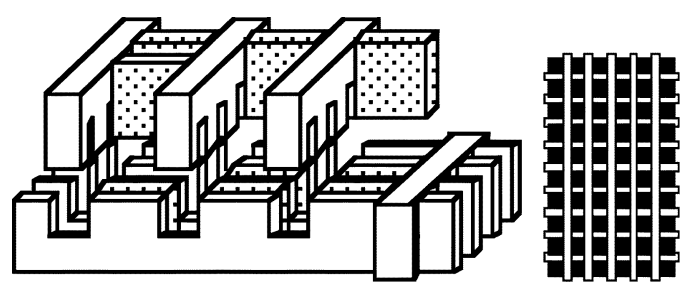

Fig. 11. One directional high $\mu$ material can be composed from magnetic material inserted in nonmagnetic separators. Right figure shows top view.

for the demonstration; a moderate one may be a piecewise linear approximation. This example also has four sets of trim coils whose currents are less than $10 \%$ of the main coil to change the field gradient. The optimization process for the step shape, coil positions and their current is briefly explained in the figure caption. The low pass filtering function makes the locality of a trim coil less significant and the field distribution becomes smooth (see Fig. 10). This turns out to reduce the number of trim coils needed to achieve a field quality at a tolerance of a flutter in $B z$ component. In Fig. 10, $\Delta \mathrm{Bz}<4$ Gauss is achieved even with the exaggerated example, where $B z$ at the center $(\mathrm{R}=500 \mathrm{~cm})$ is $0.14 \mathrm{~T}$ for the case of $\mathrm{n}=3.5$. Deviation of $\mathrm{n}$-index for this case is less than 0.1. As described before, better field distribution should be achieved with a moderate piecewise linear approximated pole surface, while it may cost higher than the stepwise case.

\section{Single EAsy AXIS Material}

The composed anisotropic material introduced and discussed in the above sections has high permeability in two directions. These directions can be reduced to only one by applying a configuration shown in Fig. 11, where the magnetic materials are
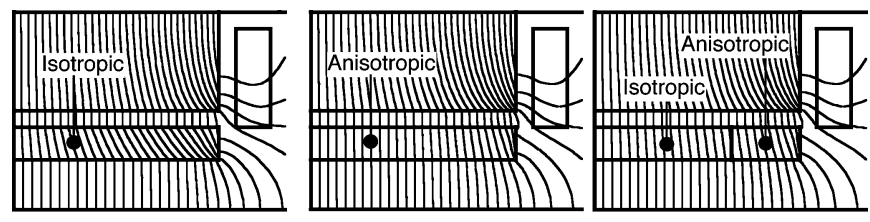

Fig. 12. Geometries to compare absolute values of the fringing field, which show the effects of the anisotropies.

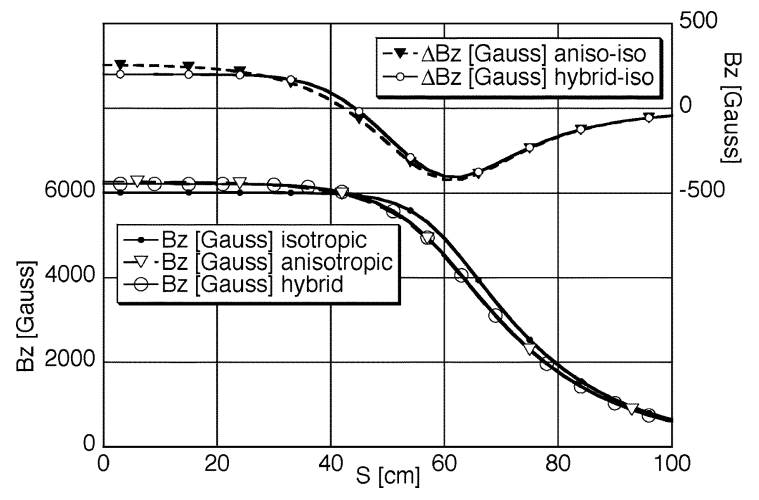

Fig. 13. Fringing fields with different anisotropies. Magnified differences from the isotropic (in the paper plane) inter-pole are also shown.

inserted in a grid made of nonmagnetic separators. Using this kind of composed material at magnet edges can reduce an absolute value of the fringing field.

Three inter-pole configurations are compared: layered material (stacked in the paper direction), single axis material (vertical) and hybrid material of these two (see Fig. 12). The first one has the same geometry as that in the bottom left of Fig. 6 where the permeability in the direction normal to the paper is supposed to be low, while the permeability in the horizontal direction is high. The second one has anisotropy in the paper plane and low permeability in the horizontal direction. This structure shows the lower fringing field but has less uniformity in the inner gap region (see Fig. 13). The third one has the anisotropic material at only the edge. This one exhibits flat distribution at inner gap region because of the isotropic inter-pole and less fringing field because of the anisotropic inter-pole. It should be noted that the field level at the inner region is higher with the anisotropic inter-pole due to a less leakage flux to the fringing area. Although just a laminated sheet material can be used as the anisotropic material for a simple dipole without any field gradient, better result will be expected if we use such unidirectional-high- $\mu$ material at the perimeter of the inter-pole. The hybrid configuration is further investigated in the following section.

\section{Higher MAGNETIC FIELD APPLICATION}

Although the smaller saturation level of the magnetic flux density due to the smaller packing factor may restrict its applications, changing the packing factor from location to location may relieve this. Usually a high field area is localized and has less field gradient, and thus less anisotropy is required. Therefore we can use solid iron in the high field area to improve the flux density and reduce the packing factor toward the low field region. Fig. 14 shows an example where the packing factor of 


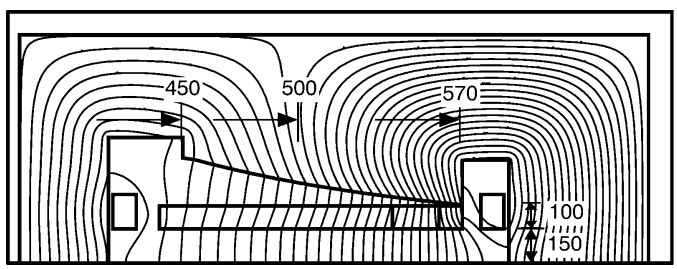

Fig. 14. The packing factor is increased toward the right edge to hold higher magnetic flux density. The $\mu_{\mathrm{e}}$ 's of the parts of the inter-pole are 3, 5 and 10, from left to right. The corresponding packing factors are $0.677,0.2$ and 0.9 , respectively.

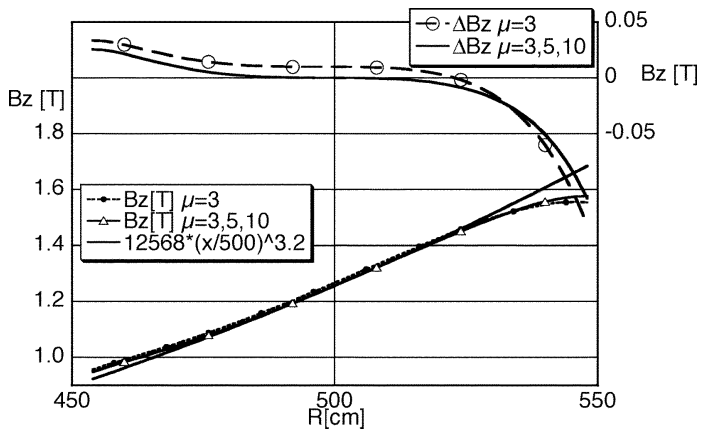

Fig. 15. Field distributions and their deviations from a power function. Broken line: with constant packing factor. Solid line: Stepped packing factor. The good field region is extended in the latter case.

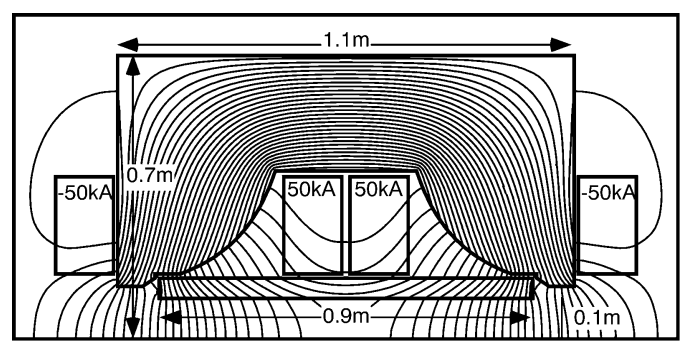

Fig. 16. A quadrupole magnet that generates quadrupole filed in a wide flat space. The packing factor of the interpole is 0.67 .

the inter-pole changes stepwise. The resulting n-index becomes -3.2 because of the higher horizontal effective permeability, while the original n-index is -3.5 . The improvement in the field distribution can be seen with the varied permeability, where the main cause of the field drop at the right is the less extent of the pole in this example).

\section{Flat Pole Q-MAGNET}

One can design a magnet that generates a quadrupole field in a wide flat space with the inter-pole as shown in Fig. 16. The effective length should be fairly constant and the extent of the stray field is also small. Fig. 17 shows the calculated field distribution of the example. The deviation of the field gradient is less than $1 \%$ over $0.6 \mathrm{~m}$ width in a $0.2 \mathrm{~m}$ gap height, where

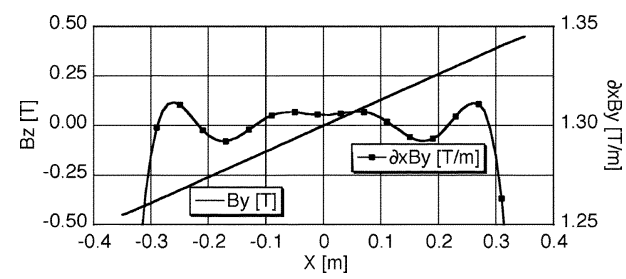

Fig. 17. Magnetic field and gradient as a function of horizontal position X. Only this example uses Cartesian-coordinates among the examples in this paper because this has wider application.

the packing factor of the inter-pole is 0.67 . Further optimization should be possible with careful choice of the parameters. Connecting the separated yokes in this example at the outer edges may ease the fabrication. This kind of quadrupole magnets should be useful for a transport system of a wide sheet beam. This type of Q-magnet can be very thin without having the contamination of higher order harmonics, which are usually caused, by large fringing fields from the complex gap changes in the transverse direction. The generated magnetic field distribution should be well defined in longitudinal direction because of the constant gap.

\section{DISCUSSIONS}

Further reduction of the fringing field is possible by using the well known field clamp, while caution is needed not to make the field clamp saturate magnetically. This sometimes happens if the field clamp is too close to the pole or there is excessive fringing field.

When we change the packing factor of the inter-pole, the actual flux density in the iron sheet may be kept constant. It should help to widen the excitation range of the magnet since the operating point of the magnetic material is uniform in the inter-pole [4]. Putting air spaces in the yoke area should achieve a uniform flux density in the yoke, which should give better result. The use of inter-poles should widen the way of designing magnets with complex magnetic field distributions.

\section{ACKNOWLEDGMENT}

The authors thank C. M. Spencer, SLAC for improving the English of their paper.

\section{REFERENCES}

[1] A. Morita, Y. Iwashita, and A. Noda et al., "Design and measurement of a combined function magnet intended for a cancer therapy accelerator," PHYS REV SPEC TOP-AC vol. 4, no. 12, art. no. 122401 Part 2, Dec. 2001.

[2] A. Morita et al., "A compact proton synchrotron with combined-function lattice dedicated for cancer therapy," in Proc. 1999 Particle Accelerator Conf., 1999, pp. 2528-2530.

[3] A. Sato, "The prism project at the high-intensity proton machine project," Nucl. Phys., vol. A721, pp. 1083-1086, 2003.

[4] Y. Iwashita, A. Noda, T. Shirai, A. Morita, M. Tadokoro, J. I. Hirota, M. Umezawa, and K. Hiramoto, "Novel design for electromagnet with wide excitation range," Jpn. J. Appl. Phys., vol. 38, pp. 895-897, 1999. 